Rev. Fac. Ing. - Univ. Tarapacá, vol. 14 No 1, 2006, pp. 49-63

\title{
BASES PARA LA CREACIÓN DE UNA METODOLOGÍA DE ADOPCIÓN DE COMERCIO ELECTRÓNICO PARA LAS PYMES CHILENAS
}

\section{AN ELECTRONIC COMMERCE ADOPTION METHODOLOGY FOR THE CHILEAN SME}

\author{
Cristian Plana $^{1}$ Narciso Cerpa $^{2}$ Per B. Bro ${ }^{3}$ \\ Recibido el 24 de marzo de 2005, aceptado el 31 de agosto de 2005 \\ Received: March 24,2005 Accepted: August 31,2005
}

\begin{abstract}
RESUMEN
Esta investigación propone una metodología de adopción de comercio electrónico, la que está enfocada a las pequeñas y medianas empresas (PYMES) Chilenas. La metodología comprende las etapas esenciales en la adecuación de éstas al comercio electrónico, por lo que se presentan elementos estratégicos en sus primeras etapas, así como, elementos funcionales y operativos para la implementación de la metodología a la PYME de una forma exitosa. Esta metodología fue validada a través de dos casos de estudio realizados en empresas PYMES que cuentan con implementaciones de comercio electrónico y que han tenido que enfrentar los problemas característicos que se presentan con la adopción de esta tecnología.
\end{abstract}

Palabras clave: PYMES, comercio electrónico, metodología, adopción, pequeñas y medianas empresas.

\section{ABSTRACT}

This study proposes a methodology for adopting e-Commerce in the Chilean small and medium enterprises (SME). This methodology includes the essential steps required to adapt the organization to e-commerce, with strategic components for the first stages, and functional and operational steps in the later stages for the successful implementation of the methodology in the SMEs. This methodology was validated through case studies undertaken in two SMEs that have implemented eCommerce, and have faced the typical problems presented with the adoption of this technology.

Keywords: SME, electronic commerce, methodology, adoption, small and medium enterprise.

\section{INTRODUCCIÓN}

El gran desafío que las empresas están enfrentando hoy, dice relación con la forma en que están adoptando y adaptando los nuevos advenimientos que se producen en el mundo de la Tecnología de la Información y Comunicación (TIC) a sus negocios [21]. En el caso de las PYMES, la adopción de las TIC induce un potencial de ahorro de costos operacionales significativos (estimado al 10\%), al tiempo que el acceso a una demanda ampliada mejora las perspectivas de ventas [17], [19]. Bajo estas circunstancias, un grupo de empresas adelantadas que acceden a estos beneficios puede hacer peligrar seriamente la posición de sus competidores que no lo hacen, e incluso, su viabilidad como proyecto empresarial.
Aquí se plantea una metodología de adopción de Comercio Electrónico (CE) enfocada a la PYME chilena, sector que preferentemente requiere de métodos que le ayuden a utilizar de forma eficiente los recursos destinados al comercio a través de Internet. La importancia de este estudio estriba en que $97 \%$ de las empresas chilenas corresponden a PYMES [18], además de entenderse que la tasa de penetración de las TIC en estas empresas alcanza un $66 \%$ de conectividad actualmente, muy superior al $42 \%$ presentado al año 2000 [18]. Cabe destacar que esta alza coincide con un desconocimiento de herramientas que permitan adecuarse a los nuevos advenimientos tecnológicos, por lo que las PYMES necesitan de apoyos metodológicos que permitan adoptar CE. Así, esta metodología comprende en su

\footnotetext{
1 Universidad de Talca, Facultad de Ciencias Empresariales, Avda. Lircay s/n, Talca, Chile, cristianplana@yahoo.com

2 Universidad de Talca, Facultad de Ingeniería, Merced 437, Curicó, Chile, ncerpa@utalca.cl

3 Universidad de Talca, Facultad de Ingeniería, Merced 437, Curicó, Chile, pbro@utalca.cl
} 
concepción modelos de adopción de CE aplicados a nivel internacional, que forman la base del diseño metodológico.

Esta investigación comprendió en primer lugar estudios sobre CE en PYMES chilenas, cuyo objetivo fue identificar la percepción y actitud de las PYMES con respecto al CE [18]. Posteriormente se analizaron modelos de adopción de $\mathrm{CE}$, clasificándolos respecto a características buscadas, además de ir identificando los factores de importancia para adecuarlos a la realidad nacional, y ser aplicados en la metodología propuesta en este artículo. Complementariamente, se analizó la evolución del CE en el país, identificando factores de crecimiento y niveles de influencia en la estructura organizacional de las empresas involucradas [18]. Una vez diseñada la propuesta metodológica, se procedió a la validación de la misma, la que comprendió un estudio de carácter teórico y práctico. La validación teórica consistió en verificar que la metodología propuesta incluye todos aquellos aspectos relativos a la adopción de nuevas tecnologías en la planificación estratégica de una empresa. La validación práctica consistió del estudio de casos de implementaciones de comercio electrónico en dos PYMES. El fin de lo anterior es permitir presentar la metodología a los entes involucrados en la toma de decisiones, de forma que sea comparable en resultados esperados, a lo que predice la teoría y práctica de la adopción de CE en PYMES.

\section{MARCO TEÓRICO}

Se denomina a las Tecnologías de la Información y Comunicación (TIC), al conjunto de avances tecnológicos que proporcionan la informática, las telecomunicaciones y las tecnologías audiovisuales. Estas tecnologías básicamente proporcionan información, herramientas para su proceso y canales de comunicación. Además, se define el CE como el uso de TIC para mejorar las comunicaciones y transacciones con todas las personas y/o organismos implicados, los cuales pueden ser clientes, proveedores, instituciones financieras, etc. [20].

Para efectos de este estudio, se define adopción de CE, como la incorporación en términos estratégicos, de tecnología de información que permita transacciones con clientes (B2C) y proveedores (B2B).

Además, se clasifican los modelos en tres enfoques distintos, complementarios entre sí. El primer enfoque se orienta al cliente/usuario de la plataforma de CE. Aquí se analiza el comportamiento del usuario, relacionado con la percepción que se tiene respecto a la seguridad online. El segundo enfoque se orienta a la empresa propiamente tal. El tercer enfoque se refiere a modelos orientados a la evolución de la plataforma de $\mathrm{CE}$, los que indican claramente funcionalidad y los elementos necesarios para la implementación de cada etapa.

\section{Enfoque-Cliente/Usuario}

Del análisis de los modelos, se distingue en primer término lo concerniente a los riesgos aceptados por parte de la PYME, principalmente las que se encuentran en posición de adoptar CE. Es de suma importancia considerar que existe un riesgo percibido, representado por parte de los usuarios que acceden a la plataforma comercial, y que este riesgo siempre está presente y afectará el comportamiento de compra del cliente virtual. De lo anterior, dentro de los modelos que se presentan, el que considera este aspecto es el Modelo de Adopción de Comercio Electrónico (e-CAM) [8].

Modelo e-CAM: Este modelo se deriva de la teoría del riesgo percibido y del modelo de aceptación de tecnología TAM [9]. El modelo e-CAM integra el modelo de aceptación de tecnología (TAM) con la teoría del riesgo percibido para explicar la adopción de comercio electrónico. Es así, como el modelo e-CAM determina, entre otros asuntos, que el comportamiento de compra se encuentra condicionado por la percepción del usuario sobre facilidad de uso de la plataforma tecnológica y, por otro lado, de la utilidad percibida de ésta (monetaria, tiempo, comodidad, confianza, etc.). Por su parte, esta utilidad está condicionada por el riesgo percibido con los productos o servicios que se ofrecen (cumplimiento de las condiciones establecidas) y por el riesgo percibido en el contexto de las transacciones (seguridad online de las transacciones que se realizan).

Del modelo e-CAM se desprende que es importante saber el porqué y el cómo los consumidores toman sus decisiones de consumo, para que la empresa pueda tomar mejores decisiones estratégicas. El objetivo es prever la forma posible en que los consumidores reaccionarán ante las señales que dan las nuevas tecnologías, particularmente en Internet. Esto último afecta principalmente a aquellas empresas que tienen un mercado potencial bien definido, dado principalmente por recursos, zona geográfica de venta; y poder comprender cómo los usuarios estimarán la presencia comercial de la empresa en Internet.

Modelo de Riesgo percibido en transacciones online: La influencia del riesgo percibido se explica con la adquisición de un compromiso por parte de un consumidor virtual con una compra, cuando éste tiene una gran confidencialidad y transparencia en la 
transacción, además de que la transacción misma se mantenga dentro de un marco legal definido [9].

De acuerdo al modelo de riesgo percibido, se identifican cuatro tipos de riesgos percibidos en el contexto de transacciones online (RTO): Privacidad, Seguridad, Irrefutabilidad, y todos los demás riesgos percibidos en una transacción online. De lo analizado para el diseño metodológico, se considera de vital importancia:

- Riesgo percibido en transacciones online: Lo más importante en la venta online es que las personas sientan confianza en una compra. De esto se infiere que el desafío que se enfrenta es brindar las herramientas que aseguren la seguridad y privacidad.

- Riesgo percibido con productos y servicios: Gran parte de las limitantes que aseguran que no se consiga una venta online es que los consumidores no observan lo que están adquiriendo. Según se evidencia, en Chile aún no existe una cultura que permita las ventas online a la par con los países desarrollados [18].

Modelo de Aceptación de Tecnología (TAM): Las empresas que no han innovado la adopción de CE deben examinar los factores que determinan la aceptación de tecnología. Existen modelos que estudian el comportamiento del consumidor, derivados de la teoría de la difusión de la Innovación de Rogers [17], los cuales analizan la percepción acerca del uso de una innovación y los efectos que conlleva. De estos estudios surge el modelo TAM adaptado que se deriva del modelo de adaptación de tecnología TAM, el cual analiza la actitud y comportamiento relacionados con la aceptación de uso de tecnología.

TAM adaptado predice la aceptación de uso basado en dos comportamientos: Facilidad de Uso Percibido y Utilidad Percibida, ambos determinantes de intención de uso individual de TIC.

Facilidad de Uso percibido: Los sistemas de información se perciben en un nivel de complejidad menor o mayor dependiendo de la probabilidad de su adopción y uso.

Utilidad Percibida: Los consumidores podrían adoptar CE, si su percepción sobre la compra online da utilidad por sobre el medio tradicional, y mejora los deseos de compra.

En resumen, lo que deriva el análisis de estos modelos, es identificar los principales factores que debe considerar una PYME en su relación comercial con clientes y/o proveedores:
- Confianza: Crear confianza en los visitantes es el mejor método para asegurar un tráfico comercial en el sitio Web, y aumentar la lealtad de los clientes. El enfoque más efectivo es entender la generación de confianza como un proceso de reducción de la incertidumbre [1].

- Seguridad y Privacidad: Para realizar transacciones tipo comercial a través de Internet, los aspectos fundamentales que se deben entender sobre los sistemas de seguridad son: confidencialidad, fiabilidad, integridad y autenticación de datos [4].

- Riesgo: Desde el punto de vista del consumidor, el que se presente un nivel de confianza aceptable en una transacción online representa el punto más importante para contrarrestar el riesgo que se percibe al hacer una compra online [8]. Hay que considerar que el consumidor online nunca se sentirá totalmente seguro al realizar una compra, ya que ésta no se realiza en el acto. Justamente, los comerciantes deberán producir confianza en el consumidor cuando exista un elevado nivel de riesgo percibido en una transacción, mientras más significativa sea una compra y más desfavorables sean los resultados percibidos en caso de que ésta fracase.

\section{Enfoque-Empresa}

Según lo referente a la empresa, los modelos de adopción estudiados comprenden factores internos y externos a ésta [13].

\section{Factores Internos}

Se debe contar con una estructura que brinde las garantías necesarias para poder tener completa seguridad de que el CE será un factor comercial importante a largo plazo [12]. Así, de acuerdo a éste modelo de adopción de CE, y tomando como referencia, asimismo, los factores organizacionales presentes en otro modelo de adopción, se derivan las siguientes conclusiones:

Primero, la incorporación de nueva tecnología debe contar con un soporte de negocio y de apoyo técnico, dado preferentemente por recursos tecnológicos actualizados (computadores, conexión constante, seguridad en las transacciones, etc.), que den a la empresa capacidad de poder desarrollarse ante un "posible" incremento constante en la venta de productos y servicios a través de la Red. Además, la importancia de disponer de personas especializadas en CE hace que la incorporación de tecnologías en la empresa se haga de acuerdo a un nivel de independencia respecto a entes externos a ésta. 
En lo referente a cómo la empresa se adecua a cualquier innovación que se presente, se debe considerar cómo la innovación modificará los hábitos que presenta una empresa que desee adoptar CE [14]. Por lo mismo, los factores que toda PYME debe considerar son los siguientes: Ventaja relativa percibida, Compatibilidad, Complejidad, Trazabilidady Observabilidad.

Así, de acuerdo a lo evidenciado en estudios de investigación a nivel nacional aquí analizados (Subsecretaría de Economía, INTEC, etc.), de los factores de innovación identificados en las PYMES chilenas, se deriva lo siguiente:

Ventaja Relativa Percibida: Este factor está relacionado con los beneficios percibidos que puede tener una empresa en el proceso de apertura de sus negocios al CE. Las PYMES chilenas perciben la utilidad del uso de Internet para lograr un mejoramiento en su negocio, referenciado en que sobre el 55\% de las PYMES lo usa básicamente para buscar información para su negocio. En cuanto al CE propiamente tal, sólo el $18,4 \%$ de las empresas reconoce la ventaja del comercio virtual para vender sus productos y/o servicios, razón aprovechable para buscar nuevas oportunidades de avanzar en calidad y productividad [18].

Complejidad: Definida aquí, como la dificultad percibida por las PYMES para adoptar el CE. Según lo precisa el Modelo de Innovación [17], la empresa que no cuenta con la capacidad suficiente para solventarse con eficiencia en el mundo de las nuevas tecnologías no estará preparada para comenzar a transar sus productos en la red. Así, se podría deducir que en la mayoría de los casos sólo el segmento de las medianas empresas puede estar preparado para tomar decisiones de adopción, dejando sólo que las pequeñas empresas usen la red para la búsqueda de información.

Compatibilidad: Definido como la capacidad de una empresa de enlazar la incorporación de Internet en su actual proceso de negocios. Las PYMES son técnicamente capaces para generar un producto o servicio y hacerlo llegar a los clientes, pero administrativamente son muy deficientes en el manejo de los recursos.

Costo: La cantidad de inversión necesaria para actuar con total independencia de entes externos a la empresa en el mundo del CE está disponible sólo para las medianas empresas, de las cuales el 100\% posee computadores, además de un alto porcentaje de conexión a Internet. Contrario a esta realidad, sobre el $40 \%$ de las pequeñas empresas no cuentan siquiera con un $\mathrm{PC}$, lo que influye en que éstas tengan que pedir ayuda a entes gubernamentales, además de verse forzadas a contratar asesorías que ayuden a vender sus productos por medio de otros intermediarios en la red.

Considerar los factores que son importantes antes de tomar una decisión de adoptar o no el CE, adquiere suma importancia de acuerdo a lo revelado por los resultados presentados en cuanto a actitud respecto al uso de nuevas TIC. Entre lo evidenciado, lo relevante expuesto es:

Primero, a pesar de que el 62,7 \% de las PYMES del país tiene computador, sólo el $41,8 \%$ de ellas tiene algún tipo de conexión a Internet, lo que revela algún tipo de falta de compromiso gerencial con la difusión de tecnologías [18]. Segundo, sólo un 12,6\% de las PYMES tiene página Web, lo que indica un notorio atraso en las políticas de desarrollo comercial de las empresas. Tercero, hay compatibilidad entre el tamaño de la empresa y el acceso a TIC, pues el nivel porcentual de computadores por empresa va en relación con el tamaño de ésta, un 15,4\% para las pequeñas empresas y un $44,1 \%$ para las medianas; lo que indica que el factor costo de acceso y recursos disponibles para la innovación es de real importancia. Por último, un $26,2 \%$ de las pequeñas-medianas empresas no sabe para qué les serviría la conexión, además de no tener los conocimientos necesarios antes de tomar una decisión de adoptar o no la nueva tecnología.

\section{Factores Externos}

Las pequeñas empresas que comienzan a comercializar sus productos en Internet son más vulnerables a la presión competitiva, mientras menor sea la cantidad de competidores. Al contrario, las medianas empresas ya tienen en gran parte asumida esta presión, mediante una apertura más decidida a las nuevas tecnologías y a una adecuación de estrategias de integración en el mercado. Por otra parte, el factor presión de proveedores se identifica en las PYMES chilenas, en que sobre el $30 \%$ de las medianas empresas se conectan a Internet para mejorar la gestión y productividad, mediante el conocimiento de que sus proveedores se conectarán [18]. Según se infiere de los resultados presentados, sobre el 55,2\% de las PYMES chilenas cotizan sus productos y/o servicios a través de Internet, lo que indica que el proceso de distribución y compra de productos a través de la red se hará más eficiente mientras se responda rápidamente a esta presión.

\section{Enfoque-Adopción e Implementación del Comercio Electrónico}

Modelo de Adopción de Comercio Electrónico eMICA: El modelo eMICA destaca que en el desarrollo de un sitio Web se construirá con el tiempo funcionalidad, 
dependiendo de cómo la empresa vaya adecuándose al uso de herramientas tecnológicas que brinda Internet [2], [5]. El modelo eMICA distingue tres etapas: Promoción, Provisión y Procesamiento.

La primera etapa, Promoción, se caracteriza, porque la empresa presenta un sitio Web que carece de funcionalidad, y sólo se presenta información de carácter visual e informativo, con escasos enlaces entre el probable cliente y la empresa. Así, el modelo eMICA propone que en esta primera etapa el sitio Web conste de información estática, la cual tiene que estar acorde a los objetivos propuestos en un plan básico de marketing, principalmente resaltar la imagen de la empresa.

En la etapa de Provisión, se añade funcionalidad al sitio Web, lo que permite agregar un sistema de información más detallado sobre los productos y/o servicios que ofrezca la empresa, con esto se puede tener el inicio de un sitio Web dinámico que permita conocer con mayor claridad para el usuario todos los recursos que tiene la empresa disponible. Aquí se identifican diferentes niveles de provisión de la información dependiendo de las características propias de la empresa. Cabe destacar que esta diferencia de niveles se da sólo con una diferencia de añadir valor a cada nivel de funcionalidad.

Nivel 1. Bajo nivel de interactividad, catálogos de productos básicos, links a información más detallada, registro en formularios online.

Nivel 2. Medio nivel de interactividad, nivel más alto de los catálogos de productos (base de datos propia), ayuda al usuario (ejemplo, mapa del sitio Web), links a otros sectores de industria, etc.

Nivel 3. Alto nivel de interactividad, salas de chat, foros de discusión, aplicaciones multimedia, y envío de noticias por e-mail.

Por último, la etapa de Procesamiento es caracterizada, porque el sitio Web alcanza una madurez funcional que permite realizar transacciones online, de modo que exista un flujo de información constante entre la empresa, sus clientes, proveedores y los socios comerciales que ésta pueda tener. Por ello, es necesaria la incorporación en el sitio Web de información que contenga elementos de seguridad, por medio de la identificación y actualización constante de las personas y/o instituciones que están presentes en una transacción.

En la siguiente sección se describirá la metodología de la investigación que se utilizó para lograr diseñar la metodología de adopción de comercio electrónico propuesta en este estudio.

\section{PROCESO DE INVESTIGACIÓN}

El estudio está basado en conceptos de adopción y difusión de las nuevas Tecnologías de la Información y Comunicación (TIC). Simultáneamente, la metodología propuesta profundiza el contexto de la planificación estratégica, y de cómo la adopción de TIC afecta la forma de transar los productos y servicios en el ambiente del CE.

Debido a que esta metodología pretende ser implementada en un sector específico de la economía nacional, se reflejan en el estudio los factores que inhiben la adopción de $\mathrm{CE}$, éstos derivados del análisis de los modelos de adopción estudiados. Estos modelos fueron elegidos de acuerdo a los objetivos buscados en el proyecto y las necesidades requeridas por la metodología. Complementariamente, se utilizó como base los resultados encontrados en una investigación sobre percepción y actitud respecto al CE a nivel nacional [18].

Dado este análisis, el objetivo que persiguió esta investigación fue proponer una metodología de adopción de CE adaptada a la pequeña y mediana empresa del país. Para este propósito, se necesitó identificar la percepción $\mathrm{y}$ actitud de las PYMES respecto al CE propiamente tal, mediante un análisis en profundidad de la situación actual, que permitiera llegar a los objetivos específicamente buscados. Esta primera etapa del estudio metodológico se basó en un estudio de información secundaria respecto al tema, abarcando varios tópicos como, por ejemplo, situación actual del CE a nivel global y local, características de las PYMES a nivel nacional, tendencias internacionales y otros.

En una segunda etapa del estudio metodológico, se analizaron modelos de adopción de CE cuyo objetivo fue estudiarlos y analizarlos, seleccionando una muestra que permitió hacer comparaciones entre ellos y la situación que se presenta en el país. Los modelos fueron estudiados en su estructura, características y alcances, identificando cada una de las partes que los componen. Dada esta situación, se procedió a la evaluación de cada uno de los modelos, basándose en la información proporcionada por el análisis primario de la situación actual del CE en Chile, y el posterior diseño de la metodología que en páginas posteriores es propuesta.

De los dos anteriores procesos metodológicos, se buscó representar en el modelo propuesto, como se debe implementar el CE y cuáles son las herramientas tecnológicas y de proceso que deben estar incluidas en el plan de adopción del mismo. 
Los pasos a seguir en el proceso de investigación (ver figura 1) son iterativos (representado por flecha con línea punteada) y buscaron representar la situación actual de modelos de adopción, las PYMES y sus requerimientos. Aquellos pasos que se encuentran en un mismo nivel se pueden ejecutar en forma paralela. En primer lugar, se busca explicar los fundamentos teóricos que inducen la incorporación exitosa del $\mathrm{CE}$ en las empresas. Este análisis teórico tiene como objetivo fundamental hacer un ordenamiento del proceso de toma de decisiones en la empresa, y adecuarlo al diseño de la futura presencia Web, o en su caso, una modificación de las estrategias funcionales que están incluidas en el diseño y desarrollo comercial virtual. En una segunda etapa, se buscó representar la implementación de estas estrategias a los cambios funcionales o de proceso que inducen esto, para ello, se complementa la primera etapa del diseño metodológico con los cambios que se presentarán, tanto internos como externos a la empresa. La idea de lo anterior es alinear los objetivos estratégicos con los funcionales.

En cuanto a la validación propiamente tal, se hizo el análisis de acuerdo a dos estados. Primero, se verificó que la misma sigue un proceso metodológico, que está acorde a lo que explica la teoría de incorporación de TIC a empresas. En forma generalizada, lo anterior se refleja en que, antes de tomar la decisión de adoptar CE, se debe considerar todo el aspecto estratégico que es materia de toma de decisiones en la empresa [7]. Además, según lo indica la teoría de implementación tecnológica, se reafirma que una empresa que adecua su presencia comercial a una virtual, debe considerar la estrategia general a sus objetivos de implementación y diseño funcional del sitio Web, es decir, los objetivos a corto y largo plazo que se buscan con esta presencia virtual [1], [15].

En segundo término, este análisis exigió comparar los resultados presentados en la realidad comercial chilena, de modo de dar viabilidad futura a la implementación de la metodología aquí propuesta. Por ello, se definió un trabajo de campo tendiente a tener resultados comparables, y entender que la metodología propuesta, es adecuada para ser presentada como apoyo teórico a la adopción de CE para las PYMES del país.

La recolección de información se realizó mediante una investigación consistente en entrevistas aplicadas a expertos en áreas funcionales de empresas que manejan el CE. Cabe destacar que para la realización de estas entrevistas se seleccionaron las empresas de acuerdo a una serie de rasgos y características estándares, tratando de observar la menor variabilidad entre sus modos de comportamiento. Por ello, este proceso exigió que las empresas tuvieran las siguientes características:

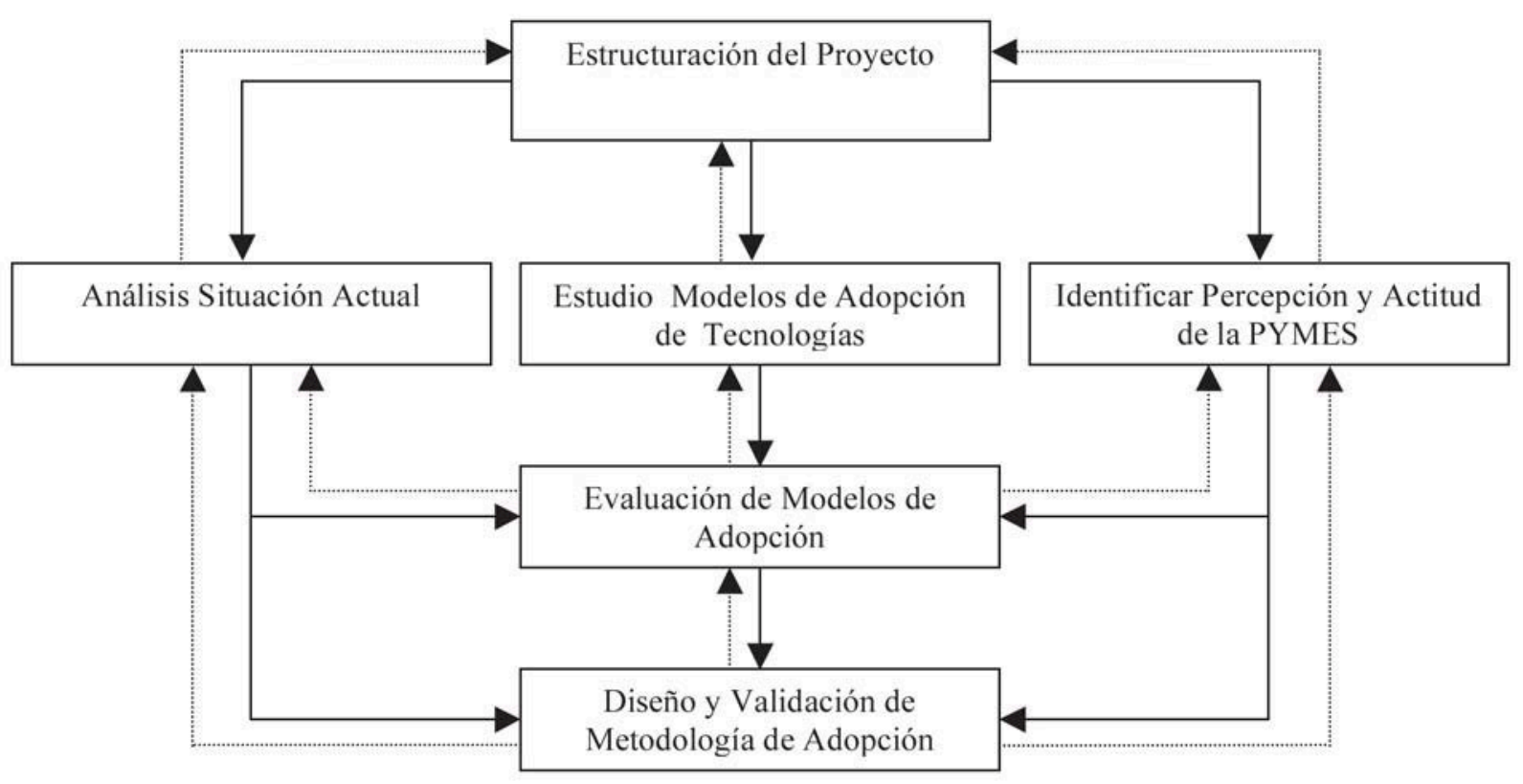

Fig. 1 Pasos a seguir en Proceso de Investigación. 
En primer lugar, las empresas estudiadas son PYMES que forman parte de la clasificación elaborada por CORFO, respecto a los distintos tipos de empresas. Además, los entrevistados han trabajado en estas empresas desde los inicios de la implementación de CE. Es decir, participaron tanto del diseño funcional como del proceso de decisión misma respecto a la adopción TIC. Por último, tienen conocimientos y experiencia suficiente respecto al tema TIC, y participan activamente en la empresa, fundamentalmente en el departamento encargado del CE.

De los resultados de estas entrevistas, se desarrollaron estudios de casos respecto a las empresas consultadas, por lo que se consiguió entender que lo propuesto por este estudio no está muy lejos de la realidad. Eso sí, se lograron descubrir falencias en materia de implementación tecnológica y ratificar lo que indicaban los estudios de investigación realizados a nivel nacional.

En la siguiente sección, se detallan los fundamentos metodológicos que explican los resultados derivados de la investigación realizada, de modo de cumplir con los objetivos buscados en este estudio.

\section{METODOLOGÍA PROPUESTA}

\section{Fundamentos}

La metodología de adopción que se presenta en este artículo servirá de apoyo preferentemente para aquellas PYMES chilenas que tengan un conocimiento sobre los factores de innovación que afectan el proceso de adopción de CE. Además, las empresas que tengan este compromiso de adopción deberán contar con recursos financieros mínimos para implementar u obtener servicios de CE.

Por lo anteriormente comentado, se presentan las derivaciones del estudio metodológico propuesto para el cumplimiento de los objetivos buscados.

En cuanto a las razones de no uso de CE, los aspectos relevantes a considerar, según lo propuesto en esta metodología, estriban en que gran parte de las empresas que están actualmente con alguna posición en la Red (o no), no están descubriendo los beneficios del CE a todo nivel de negocios, principalmente por los siguientes motivos:

Bajo apoyo por parte de los directivos, lo que conlleva a no tener el CE posicionado como una oportunidad de negocios a corto y largo plazo. Esto se refleja en que se desconocen en la mayoría de las PYMES las ventajas relativas que puede traer el CE para su negocio.
En la mayor parte de las PYMES, en especial en el segmento de las medianas-pequeñas empresas, hay una falta de visión estratégica. Esto coincide con una evidente resistencia al cambio en la forma de hacer negocios, cuando se les ofrece implementar el CE. Por último, y muy relacionado a lo anterior, existe una escasez de personal capacitado para el área específica del CE.

Según lo analizado, respecto a las necesidades de las PYMES en el ambiente del CE, y una vez realizado el análisis de los enfoques de adopción que se derivan de los modelos, se infieren las siguientes conclusiones generales, en ámbito planificación estratégica y $\mathrm{CE}$ :

- Clara definición de los objetivos del negocio, tanto en el ámbito estratégico como operativo. Definiendo políticas y procedimientos, es decir, estas definiciones deben estar planteadas por la dirección de la empresa.

- Contar con total seguridad del apoyo y creencia de los gerentes y/o altos directivos de la utilización del $\mathrm{CE}$, es decir, debe haber un apoyo enfocado a una formación técnica sobre cómo utilizar Internet para fomentar nuevos negocios a todos los niveles de la empresa.

- Apoyo de las unidades estratégicas de negocio para el proyecto de adopción de CE.

- Definir la implementación del CE como asunto de relevancia estratégica para la empresa.

- Mantenerse preparado para las oportunidades y amenazas que trae el CE, mediante una abierta disposición -a todo nivel de negocio- reflejado en un replanteamiento del modelo de negocios y los procesos mismos de la empresa.

Dado que la relación entre empresa, proveedores y clientes está significativamente afectada por la adopción de CE, la PYME debe considerar, en primer término, asegurar la generación de confianza y transparencia en el proceso; para ello se deben presentar la seguridad y privacidad en las transacciones con los clientes como aspecto prioritario en la presencia comercial virtual. Además de enfatizar en el factor riesgo percibido en toda transacción online, por parte de los que visitarán el sitio Web comercial.

Según la evidencia disponible, en la evolución de un sitio Web comercial deben considerarse los siguientes aspectos relativos a la seguridad en la red:

- Proteger la integridad del sitio Web.

- Garantizar la integridad de los datos.

- Se debe proteger el contenido de los datos distribuidos por Internet. 
- Debe resguardarse y retener la propiedad de los materiales presentes en el sitio Web.

Los elementos esenciales en relación con el proceso de adopción de $\mathrm{CE}$ referentes a materia de seguridad y confidencialidad electrónica son:

Autenticación: Se refiere a establecer la identidad de un usuario, es decir, la exigencia de validar el acceso a información de carácter personal y confidencial.

Autorización: La autorización se refiere a establecer que el usuario ya identificado tiene derecho a acceder a información específica o servicios que requieran.

Irrefutabilidad (Integridad): Esto implica garantizar que la modificación de la información de los programas se realice únicamente de la manera especificada y autorizada, y que los datos presentados no serán eliminados en tránsito [1].

\section{Niveles de Incorporación de Comercio Electrónico en la Empresa}

Se propone un modelo de adopción de CE basado en los niveles de funcionalidad que tendrá un sitio Web, éste de acuerdo a las fases que incurre una empresa para estabilizar una presencia comercial en la red. El modelo eMICA destaca que en el desarrollo de un sitio Web se construirá con el tiempo funcionalidad, dependiendo de cómo la empresa vaya adecuándose al uso de las herramientas tecnológicas que brinda Internet. En este proceso, se distinguen tres etapas [2], [5]:

1. Creación de un sitio Web.

2. Automatización del proceso de negocios.

3. Integración del Comercio Electrónico en la Empresa.

\section{Creación de un Sitio Web}

El primer paso requerido para establecerse en el CE, según lo afirma Kalakota [10], [11], es que la empresa comience por una etapa de Experimentación, es decir, proponer una presencia virtual. En cuanto a las características del sitio Web son preferentemente su carácter informativo y presencial, lo que implica que la funcionalidad asociada en esta etapa generalmente está dada por:

- Un sitio Web con limitada funcionalidad (presencia estática), que incluye información sobre la empresa en general, ubicación, forma de contactarse con ésta.

- Folletos informativos sobre productos y/o servicios ofrecidos, forma de adquirirlos.
- Escasez de links con otros sitios Web de interés.

- La navegabilidad del sitio Web es de carácter simple.

\section{Automatización del proceso de negocios}

Etapa en la que se comienzan a integrar los negocios a la red, mediante una apertura comercial del proceso de compra y venta de productos. La funcionalidad que se le agrega al sitio Web se incrementa, dado que es necesario contar con elementos de acceso y resguardo de datos, mediante la inclusión de formularios de ingreso de información y bases de datos asociadas.

En esta etapa de comienzo de una automatización del proceso de negocios, debe desarrollarse la visión de una completa integración de los negocios a la red, mediante la aplicación de lo ya planificado cuando se toma la decisión de crear un sitio Web comercial, junto con una visión estratégica de esta adopción.

A medida que la empresa se adecue a la presencia en la Web y readecue su proceso de negocios a una apertura comercial (dada por realizar una estrategia de ventas y compras online), debe considerar necesario contar con requerimientos imperiosos para actuar con eficiencia en el CE. Dentro de las características de este nivel de adopción de $\mathrm{CE}$, se cuenta lo siguiente:

- Un beneficio comercial a gran parte de las unidades de la empresa. Además de una mayor exigencia a todo nivel, debido a que se integran al comercio virtual elementos de funcionalidad y transacciones que se hacían anteriormente de forma tradicional.

- Una transferencia de información que requiere necesariamente de una base de datos segura y flexible, a los requerimientos que se hagan necesarios mientras la empresa avance en la puesta en marcha de esta adopción de CE.

- Comienzo de una automatización de proceso de negocios, lo que afectará gran parte de las unidades comerciales de la empresa.

- Aparición de nuevos intermediarios y conflictos en los canales de distribución.

- Inclusión obligatoria de elementos de seguridad en el sitio Web.

\section{Integración del Comercio Electrónico en la Empresa}

Una vez establecidas las etapas precedentes, la empresa ya está en capacidad de poder realizar una integración total de los negocios a la red. Etapa caracterizada por una completa automatización e integración del proceso de negocios, aplicando elementos de integración de los clientes (CRM), e integración de los servicios de entrega 
a domicilio de los productos adquiridos por el usuario. Según lo expone el modelo eMICA, la tecnología presente en las plataformas virtuales alcanza una madurez, que permite realizar transacciones con total seguridad.

Por ello la evolución que se sigue en esta etapa está dada por [6]:

- Aparición de mayor funcionalidad en el sitio Web, pasando de un estado de presentación estática a una de aspecto dinámica.

- Transferencia de información que requiere necesariamente de una base de datos segura y flexible, a los requerimientos que se hagan necesarios mientras la empresa avance en la puesta en marcha de esta adopción de CE.

- Comienzo de una automatización del proceso de negocios, lo que afectará gran parte de las unidades comerciales de la empresa.

- Inclusión obligatoria de elementos de seguridad en el sitio Web.

\section{Etapas y Pasos de la Metodología Propuesta}

La secuencia de pasos metodológicos a considerar para tener una adopción eficiente del CE son los siguientes:

1. Conocimiento de los altos directivos y gerentes de la Empresa sobre el tema CE, así como la definición de la actual presencia que tiene la empresa respecto a la Tecnología de la Información y Comunicación.

2. Considerar los factores que afectan todo proceso de innovación tecnológica. Aquí es importante hacer una revisión de los principales elementos afectados, tanto internos como externos a la empresa.

3. El plan estratégico de la empresa debe revisarse y adecuarse a la incorporación de Internet como un nuevo elemento de negocios, lo que exige una revisión de los recursos y la cultura de la empresa.

4. Planificación de la imagen de la empresa en la Web, dada por la presentación del sitio Web comercial respecto a una revisión constante de los elementos vistos en los puntos anteriores (pasos 1 a 3 ).

5. Analizar las necesidades de información que son requeridas para el proceso de adecuación de toda la estructura de la empresa al CE, lo que exige un estudio de los principales sistemas de información existentes en el mercado.

6. Aplicar los elementos y herramientas que permitan una disminución del riesgo percibido en materia de transacciones online, así como los elementos de seguridad que permitan afrontar con plena confianza la integración de Internet a los negocios de la empresa.

7. Reestructuración de la empresa a todo nivel de negocios, e integración temporalizada en los procesos de la empresa. Aquí se aplica reingeniería constante de los procesos de la empresa.

8. Promover una presencia en Internet comercial (transaccional) e integración real de esta presencia virtual. Análisis y aplicación del ciclo de vida cliente-proveedor.

9. Seguimiento de los pasos anteriormente nombrados, mediante un control aplicado con una retroalimentación constante de este proceso de adopción.

La metodología propuesta se enmarca fundamentalmente en un contexto estratégico que fomenta parte del proceso de adopción de CE. Es por ello que en la búsqueda del diseño de la misma se consideró la información resultante de diversos estudios sobre adopción de TIC, y de cómo ésta beneficia al contexto general de la empresa. Además, la metodología incorpora estos modelos como base para la creación de la misma, y representar los pasos tanto estratégicos como operativos que se requieren en esta adopción.

Así, se estudió la contribución de cada modelo al proceso de adopción, por lo que se clasificaron éstos en tres diversos contextos: estratégico, funcional y de ámbito operativo.

En el ámbito estratégico, se buscó identificar los factores que influyen en la adopción de CE, identificando tipos de factores considerados relevantes en la adopción y difusión del CE. Algunos de estos factores llegan a ser más importantes dependiendo del tiempo que transcurra entre la decisión de las organizaciones de adoptar esta tecnología, y el tiempo en que el CE ya ha sido implementado dentro de la organización. Todos estos factores fueron identificados dentro de dos categorías: factores de contexto internos y externos relativos a la organización. Esta distinción es propuesta para distinguir entre factores de organizaciones específicas y factores que son impuestos desde fuera de la organización, en la decisión de adopción y utilización del CE.

En cuanto al nivel funcional, los elementos considerados son aquellos que podrían requerirse para diseñar e implementar una plataforma de $\mathrm{CE}$ del tipo $\mathrm{B} 2 \mathrm{C}$, por ejemplo, identificación de lo que afecta el comportamiento de los usuarios online y de los cambios funcionales que ocurren en la organización cuando se implementa el CE. Dentro de este contexto, la 
contribución de los modelos e-CAM y TAM fueron esenciales para diseñar la metodología propuesta.

Por último, se requería obtener una metodología que permitiera la adopción gradual de $\mathrm{CE}$ en el tiempo. Por lo que se estimó como esencial para este propósito el modelo eMICA, que propone la adecuación gradual de los requerimientos del negocio al nivel operativo a través de la incorporación de funcionalidad, y determina la presencia Web de la organización.

De lo anteriormente comentado, desde un punto de vista netamente innovador, la metodología sigue un proceso evolutivo que contiene las bases teóricas descritas por Rogers [17] en su modelo de innovación y difusión de la innovación, y que son las bases generales de la metodología de adopción de CE por nuestro estudio propuesto.

Así, dado que la metodología contiene como base un análisis estratégico en el desarrollo de la presencia comercial de las empresas en la Web, y basado en los componentes de cada modelo aquí estudiado, se propone identificarla como e-STAI, dado que sigue un estudio de secuencial de carácter estratégico, seguido por una automatización de los procesos y una adecuación e integración de las decisiones en las primeras etapas de la metodología señaladas.

\section{Validación de Metodología de Adopción Propuesta}

La validación de la metodología siguió un proceso riguroso, basado en un estudio de tipo exploratorio que permitió identificar y seleccionar cuáles aspectos empíricos del problema resultaron ser más relevantes desde un punto de vista teórico y práctico. En general, para que la metodología propuesta en nuestro estudio sea representativa de la realidad nacional se enfatizó en los siguientes aspectos:

1. Validación referente a la teoría aplicada

2. Validación referente a la adecuación de la metodología a las empresas.

Tanto la validación teórica como la práctica tuvieron el propósito de verificar si las etapas y pasos de la metodología de adopción de CE propuesta en este artículo eran aplicables en las PYMES chilenas y si contribuían a la adopción gradual y segura de CE por las PYMES.

En la validación teórica, se considera todo lo relativo a la planificación estratégica de la empresa. De acuerdo a lo expuesto, antes de decidir adoptar CE, debe considerarse todo el aspecto estratégico que es materia de toma de decisiones [7]. Así, se evidencia que toda adopción tecnológica debe contar con apoyo directivo, pues esta formación sirve como un elemento motivador hacia el proyecto, ya que si el equipo directivo no está motivado, difícilmente se va a conseguir llegar a los objetivos deseados.

En cuanto a la decisión misma de adopción, se destaca el hecho que, una vez que la alta dirección adquiera conciencia que la innovación dará buenos resultados en la empresa, y se logran descubrir los beneficios de una presencia comercial en Internet, es posible considerar integrarlo a la estrategia general de la empresa [16]. Por ello, debe revisarse el plan estratégico, donde es necesario que las organizaciones cuenten con novedosos sistemas de gestión de la información, que les permitan transformar datos en conocimiento de valor estratégico para sus operaciones.

De acuerdo a lo reflejado en la metodología propuesta, el análisis interno que comprende desde la planificación estratégica de la empresa (figura 2, pasos 1 a 3), pasando por elementos de presentación de ésta en la Web, en lo que respecta a la funcionalidad que se le dará al sitio Web y las herramientas necesarias para esta adopción de CE (figura 2, pasos 4 y 5), debe ser enlazado, según lo evidencia parte de la teoría [19], con una posterior revisión en todo lo referido a elementos que afectan la percepción y actitud de los clientes/usuarios, respecto a los riegos inherentes que se fijarán éstos al momento de tener confianza y seguridad necesaria para adquirir productos y/o servicios online (figura 2, paso 6).

Para finalizar, la integración de los procesos antes descritos se ve realizada por una digitalización de la venta online, lo que incluye tratamiento sobre herramientas que permiten esto. La reorganización de los procesos se refleja en Internet para la empresa en procesos tan vitales como el ingreso de órdenes de pedido, informes de inventario, estado del pedido y soporte del cliente. Estos asuntos dependen de la capacidad funcional del sitio Web para tratar la información de una manera que otorgue beneficios comerciales a la empresa.

Según la evidencia, una vez que la empresa adecua su estrategia comercial (figura 2, pasos 1 a 3), analiza los factores que son mitigantes de la adopción del CE, y estudia a fondo los elementos necesarios para la integración comercial, se está en capacidad de fomentar un proceso de integración de nuevas tecnologías [3].

En cuanto a la validación práctica, se realizó un estudio de casos de implementación de comercio electrónico 
en dos PYMES. El objetivo de estudiar estos casos de implementación de comercio electrónico fue verificar que la metodología propuesta en este estudio es adecuada para ser presentada como apoyo teórico a la adopción de CE para las PYMES del país.

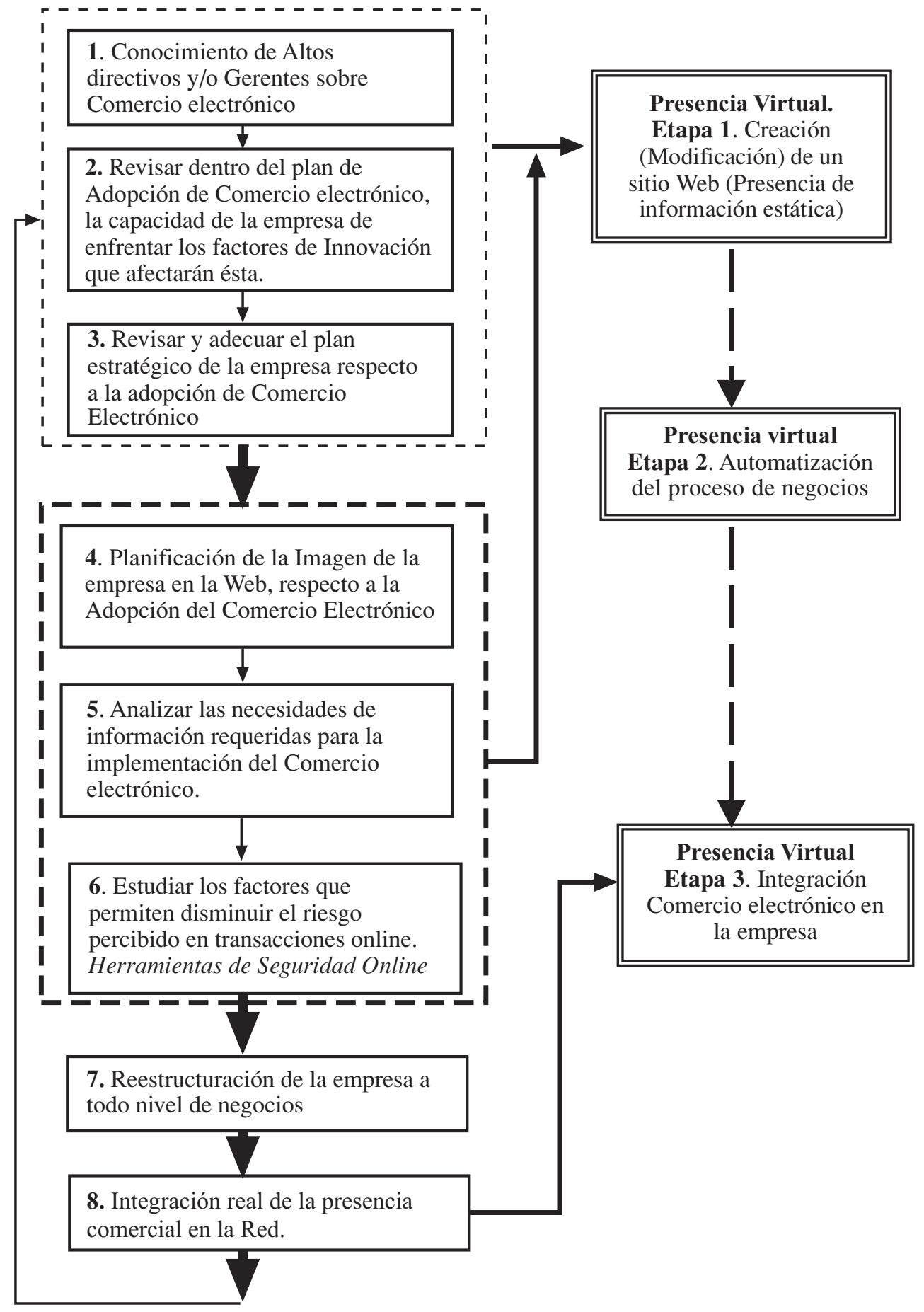

Fig. 2 e-STAI: Metodología propuesta para la adopción de Comercio Electrónico en las PYMES chilenas. 
La recolección de información se realizó mediante una investigación consistente en entrevistas realizadas con expertos en comercio electrónico de las empresas estudiadas. Cabe destacar, que dada la característica técnica de este estudio, y las limitantes propias de la situación actual de integración del CE en las PYMES del país, se seleccionaron empresas que estaban en un nivel de adecuación de CE que las hacía viables para ser incorporadas en este estudio. Considerando esto, la recolección de información consideró dos empresas que poseen presencia comercial en Internet y que han vivido una experiencia importante en la implementación de CE. Por una parte, una librería que incluye entre sus prácticas la venta por catálogos y distribución "Just in Time" de los productos adquiridos por el usuario. Esta empresa planea a futuro una integración total de sus departamentos al CE propiamente tal. Además, una prestigiosa multitienda que, si bien no está en competencia directa con las grandes tiendas del país, posee un departamento propio de CE que diseñó y desarrolló su propia presencia virtual, y está catalogada como una de las pioneras a nivel nacional (estatus PYME) en la venta y adquisición de productos online.

La entrevista a las personas encargadas de los departamentos de informática -y que a su vez son los encargados del $\mathrm{CE}$ - consistió en preguntas abiertas tendientes a abarcar la mayor cantidad de información posible, mediante la grabación de la misma. Por tanto, el formato de respuestas es "abierto", donde el entrevistado se expresó con la mayor libertad posible sobre los temas tratados.

Las preguntas realizadas abarcaron los siguientes tópicos:

En primer lugar, lo referente al proceso de planificación estratégica, en el que se consideraron aspectos relativos a análisis FODA, ventajas competitivas, y la visión estratégica que tiene la alta dirección de la empresa respecto a la adopción de nuevas TIC.

Además, se consultó sobre el diseño e implementación del sitio Web, y la forma en que éste se integró en los procesos de la empresa. Por último, se preguntó lo relativo a la funcionalidad del sitio Web y cambios requeridos en los procesos de la empresa. Esto nos permite verificar si lo realizado por las empresas estudiadas tiene alguna relación con lo que se propone como apoyo metodológico y teórico para ser aplicado en las PYMES chilenas.

Cabe destacar que las preguntas fueron tratadas mediante la indicación de áreas de conocimiento general de la empresa y las metas propias que el departamento encargado se planteó al decidirse por la adopción de
Comercio Electrónico. Así, este proceso de entrevista fue nivelado siguiendo nuestra propuesta metodológica, la que está configurada mediante las siguientes áreas de decisión comercial:

Planificación estratégica de la empresa. Ambas empresas estudiadas fueron deficientes en la planificación de la presencia virtual de la empresa, ya que ésta no fue considerada en sus planes estratégicos. Cuando fueron consultados si se realizó una planificación de la presencia virtual de la empresa, fueron conscientes en debatir que los bajos niveles de venta online que actualmente poseen se debe en gran medida a los bajos estándares de análisis comercial y compromiso gerencial con las nuevas tecnologías. Por lo mismo, debido la causa experimental de este proceso para ellos (de adoptar CE), obviaron privilegiar una presencia comercial virtual como nueva área de ventas en vez de realizar un eficiente análisis de planificación estratégica. Ejemplo de esto es que las empresas consultadas ni siquiera realizaron un análisis FODA y cómo afecta la adopción del CE a las distintas áreas de la empresa. Parte de este error estratégico ha tenido que ir solucionándose en la actualidad mediante una retroalimentación de la metodología de adopción usada por ellos, lo que influye en pérdidas de eficiencia y daños económicos que no han hecho rentable la presencia virtual de la empresa.

Los resultados de la entrevista en este ítem se evaluaron mediante la capacidad de las empresas del mismo rubro de sostener estratégicamente la adopción de CE, y de cómo la metodología aquí propuesta es viable para ser implementada en estas empresas. En general, se puede inferir que ambas empresas tienen un bajo conocimiento estratégico de los pasos esenciales en toda implementación tecnológica, esto reflejado en que existe un desconocimiento de los modelos de adopción tratados en nuestro estudio, encontrándose en éstas que la integración de decisiones estratégicas y funcionales no se reconoce en los modelos.

Diseño e Implementación del Sitio Web. Lo esencial de este proceso fue comparar los pasos de nuestra metodología con la teoría usada por estas empresas para la adopción inicial del CE. Si bien en ambas empresas fueron utilizadas adecuadamente las herramientas para crear la funcionalidad a los requerimientos de diseño y procesos, esto no siguió un proceso metodológico estudiado, lo que repercutió en que se encuentren a lo largo del tiempo fallas de funcionalidad específicas que han retrasado la integración comercial de las empresas al mercado regional. Esto en parte debido a que el departamento de $\mathrm{CE}$ de ambas no tiene un plan de integración a todo nivel de negocio de la presencia virtual, 
lo que es notoria deficiencia para proyectar una integración total de la misma. Así, y de acuerdo a lo que se refiere al nexo que existió entre esta funcionalidad y la planificación de la misma -en lo referido a decisiones estratégicas por parte de la empresa- no se evaluaron estos temas en los inicios de esta adopción, por lo que consideramos como esencial para el conocimiento de este proceso integrar en la modificación futura del sitio Web elementos detallados en nuestra propuesta metodológica. Las principales conclusiones son:

- El diseño del sitio Web fue elaborado por personal de la empresa (interno).

- No se elaboró la funcionalidad del sitio Web para transar productos desde el comienzo de la presencia virtual de la empresa.

- No se estudió la teoría de adopción tendiente a diseñar la funcionalidad del sitio Web, en relación con la estrategia de la empresa.

Funcionalidad del sitio Web y cambios en los procesos. Las empresas consultadas integran generalmente elementos que están incorporados en nuestra metodología para la funcionalidad propia del sitio Web. En cuanto a los temas de seguridad online, ambas empresas responden de forma general a los elementos recomendados en nuestra metodología. Lo que cabe entender es que la funcionalidad misma es apropiada a los requerimientos por ellos buscados. En sí, esta aceptación no reconoce los lineamientos futuros de desarrollo virtual de la empresa, por lo que se ve una conformidad con lo que actualmente se realiza en ella. Estas empresas poseen elementos básicos de seguridad, que son destacados en niveles primarios de la adecuación del sitio Web al CE (integridad del sitio, integridad de datos). No obstante, en cuanto a la personalización de la información, hay un conocimiento básico sobre el tema, y no se reconoce como esencial en éstas lo relacionado al CRM (Customer Relationship Management). Con esto, se reafirma la posición de nuestra propuesta metodológica, al no incorporar en estas empresas este elemento esencial para afianzar un conocimiento más adecuado de los clientes y que dé beneficios aceptables en estas empresas.

En resumen, la generalización de la funcionalidad está conforme a lo destacado por nuestra metodología. Si bien, en la práctica no se puede concluir que nuestra metodología es aceptable en niveles más desarrollados de CE, se reafirma en niveles básicos de funcionalidad que los elementos buscados por toda empresa son los mismos que se representan en nuestra metodología, y forman herramientas básicas para el diseño de una metodología en particular propuesta. No obstante lo anterior, consideramos práctica y teóricamente esencial considerar nuestra metodología para actuales estudios de adopción de CE.

Los tres elementos anteriormente comentados y analizados en cada empresa son aceptables desde el punto de vista teórico, dado que nuestra metodología integra elementos básicos conocidos por una empresa que tenga decidido desarrollar el CE en su negocio. Aun así, cada una de ellas buscó pasos propios para adecuarse al CE, que no son reconocidos por nuestra metodología en el estudio realizado. La falta de una metodología podría explicar por qué estas empresas están actualmente estancadas y no han podido desarrollarse virtualmente en la forma deseada. No existió en ninguno de los casos una planificación estratégica acorde a los requerimientos actuales de adopción de Comercio Electrónico.

\section{CONCLUSIONES Y RECOMENDACIONES}

Dentro del análisis que reveló este estudio, las principales conclusiones están enfocadas a representar cómo el plan estratégico de cada empresa es un gran influyente en la incorporación de TIC, y de como la tecnología debe adecuarse a cada empresa, según las necesidades de la misma.

En la metodología propuesta, cada paso está representado como un proceso de decisiones, en el que la sucesión en el tiempo de este proceso depende de la estrategia a seguir y la implementación funcional del sitio Web. Por lo mismo, el fin de la adopción de CE es buscar el mayor grado de automatización esperado, para poder interactuar en la transformación de datos a información de carácter estratégico para las empresas. A su vez, la estructura metodológica sigue un dinamismo funcional, que permite entender los principales lineamientos estratégicos y características necesarias, para nivelar la capacidad que tiene cada empresa en la evaluación de cada paso. Se entiende esto en que el modelo sigue una transición que va de una presencia estática en la Web a una de carácter dinámica, donde están integrados los sistemas de información dentro de la empresa.

Por lo anteriormente comentado, las conclusiones se pueden representar mediante los siguientes asuntos: planeación estratégica, implementación tecnológica y gestión de la información.

Según lo inferido por parte del presente estudio, las PYMES por lo general no conceden la importancia necesaria en cuanto a la planeación estratégica, esto se debe a que aún no existe una cultura innovadora, lo que 
influye en la capacidad que tengan las empresas para interactuar con niveles de desarrollo socioeconómico presentes en otros países. En la mayoría de los casos, las PYMES chilenas apuestan su presencia virtual a un carácter netamente informativo y no lo ven como una integración real de las transacciones virtuales a los negocios, lo que retrasa la incorporación de estas al escenario comercial mundial.

En cuanto a la implementación tecnológica, existe un desconocimiento de las tecnologías disponibles en el mercado, junto con una escasez de técnicos especializados en el área. Lo anterior es un impedimento para desarrollar la adopción del CE de manera masiva a nivel nacional, y con esto acercarse a los índices de adopción tecnológica que presentan actualmente países con los cuales estamos empezando a lograr estrechos lazos comerciales.

Así, considerando los factores que permiten la evolución de la presencia virtual, se detallan en la metodología propuesta, elementos de integración comercial a todo nivel de negocios. Esta integración, se especificó detallando los elementos de mayor importancia, con el objetivo de enfocar el diseño metodológico a un ámbito netamente comercial. No obstante, se destacan algunas de las herramientas tecnológicas más importantes para hacer una integración eficiente y eficaz, considerando esperar obtener beneficios de esta adopción a largo plazo.

La gestión de la información es de vital importancia en el análisis propuesto en este estudio, ya que se presenta como parte esencial de la metodología propuesta. En ella se destacan elementos de manejo de información y conocimientos de las mejores herramientas de gestión aplicables al ámbito del CE. Así, un buen manejo de la información permitirá que la empresa sea capaz de enfrentar con madurez los advenimientos tecnológicos que se presentan. Esta madurez se alcanzará sólo si se gestiona la información basado en un análisis profundo de la teoría de innovación y una puesta en práctica -mediante una actualización constante- de los conocimientos que la empresa vaya adquiriendo a lo largo del tiempo.

Gran parte del crecimiento que puedan tener las PYMES en el CE propiamente tal debe ser fomentado por organismos de ayuda a empresas, que prioricen programas de adopción tecnología, a la vez que exista una mayor comunicación entre estos organismos y las empresas que tengan la capacidad de poder solventarse en estos nuevos mercados.

Dado el carácter teórico del presente trabajo, el mismo está enfocado a servir de apoyo metodológico para los organismos de apoyo a la PYME, por lo que éstos deben profundizar los temas aquí tratados. Así, el objetivo futuro de esta investigación es servir de base para siguientes estudios de adopción del CE y entregar las herramientas necesarias a las empresas interesadas en la evolución comercial.

Resumiendo, las empresas deben contar con la mayor cantidad de información teórica posible para integrarse al CE, y se entiende a lo largo del estudio que el carácter con el que se maneja la información y la teoría aquí presentada es de vital importancia para dar un avance en políticas de apertura comercial en empresas que ven la innovación tecnológica como fuente de desarrollo y futuras ventajas comerciales.

\section{REFERENCIAS}

[1] D. Amor. "E-Business Revolution". Primera Edición. Editorial MacGraw-Hill, 1998.

[2] L. Burgess y J. Cooper. "MICA: Model of Internet Commerce Adoption". In S.M. Rahman and M.S. Raisinghani (eds). Electronic Commerce: Opportunities and Challenges. Idea Group Publishing. Hershey, USA. 2000.

[3] T. Clayton y C. Criscuolo. "e-Commerce and Business Change". Economic Trends. Vol. 583, pp. 62-69. 2002.

[4] R. del Águila. "Comercio electrónico y estrategia empresarial". Segunda Edición. Ediciones Alfaomega. 2001.

[5] B. Doolin, L. Burgess, J. Cooper y C. Alcock. "Internet Commerce Adoption by New Zealand and Australian Regional Tourism Organizations: A Comparative Study Using eMICA". Proceedings ECECR-4. Dallas. Texas. November, pp. 8-11. 2001.

[6] A. Hartman y J. Sifonis. "Net Ready. Estrategias para el éxito en la Economía". Primera edición. Editorial McGraw-Hill. 2000.

[7] M. Hitt y R. Ireland. "Administración estratégica. Competitividad y Conceptos de Globalización". Tercera Edición. Thompson editors. 1998.

[8] D. Lee, J. Park y B.S. Ahn. "On the explanation of factors affecting: E-Commerce Adoption". Proceedings of the $22^{\text {nd }}$ International Conference on Information Systems (ICIS 2001). New Orleans. Louisiana, USA, pp. 109-120. 2001. 
[9] J. Jacoby and L. Kaplan. "The Components of Perceived Risk". Proceedings from Third Annual Conference. Association for Consumer Research. University of Chicago. Chicago, Estados Unidos, pp. 382-393. 1972.

[10] R. Kalakota y A.B. Whinston. "Frontiers of Commerce Electronic". Addison Wesley. 1996.

[11] R. Kalakota. "Digitalización de los procesos en las PYMES: de la visión a la ejecución". Plenario VII Jornada EuroEcom 2BDigital: Internet como herramienta de negocio en la empresa. 2000.

[12] G. Larsen. "Security issues related to industry's use of the Internet". In Proceedings of the Association for Information Systems 2nd Americas on Information Systems. August 1720. Phoenix, Arizona, pp. 146-148. 1996.

[13] C. Ling. "Model Factors Influences on Electronic Commerce Adoption and Diffusion in Small-\& Medium-sized Enterprises". Doctoral Consortium of the $9^{\text {th }}$ European Conference on Information Systems. Bled. Slovenia. 2001.

[14] N. Rashid y Al-Qirim. "E-Commerce Technology Adoption Framework by New Zealand Small to Medium Size Enterprises". Res. Lett. Inf. Math. Sci. Vol. 2, pp. 63-70. 2001.
[15] D. Papakiriakopoulos, A. Poulymenakou A. y G. Doukidis. "Building e-Business Models: An Analytical Framework and Development Guidelines". In Proceedings of 14th Bled Electronic Commerce Conference. June 25-26. Bled, Slovenia. 2001.

[16] J. Rayport y B. Jaworsky. "E-Commerce". Primera edición. Mc Graw Hill. 2003.

[17] E.M. Rogers. "Difusión of Innovations". Quinta edición. Free Press. 2003.

[18] Subsecretaría de Economía. Encuesta "Acceso y uso de Tecnologías de la Información en las empresas chilenas". Santiago, Chile. 2002.

[19] V. Swaminathan, E. Lepkowska-White y B.P. Rao. "Browser or buyers in Cyberspace? An Investigation of factors influencing Electronic Exchange". Journal of Computer-Mediated Communication. Vol. 5. № 2. December 1999.

[20] S. Vandermerwe. "E-Technology - Fuelling or Customer Strategies?" Strategy, pp. 16-17, mayo 2000.

[21] R. Watson. "Electronic Commerce: The Strategic Perspective", Harcourt Brace College Publisher. USA. 1998. 